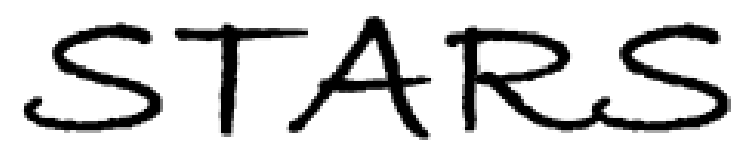

University of Central Florida

STARS

Faculty Bibliography 2000s

Faculty Bibliography

$1-1-2004$

\title{
Electrically tunable liquid-crystal photonic crystal fiber
}

Fang Du

University of Central Florida

Yan-Qing Lu

University of Central Florida

Shin-Tson Wu

University of Central Florida

Find similar works at: https://stars.library.ucf.edu/facultybib2000

University of Central Florida Libraries http://library.ucf.edu

This Article is brought to you for free and open access by the Faculty Bibliography at STARS. It has been accepted for inclusion in Faculty Bibliography 2000 s by an authorized administrator of STARS. For more information, please contact STARS@ucf.edu.

\section{Recommended Citation}

Du, Fang; Lu, Yan-Qing; and Wu, Shin-Tson, "Electrically tunable liquid-crystal photonic crystal fiber" (2004). Faculty Bibliography 2000s. 4319.

https://stars.library.ucf.edu/facultybib2000/4319

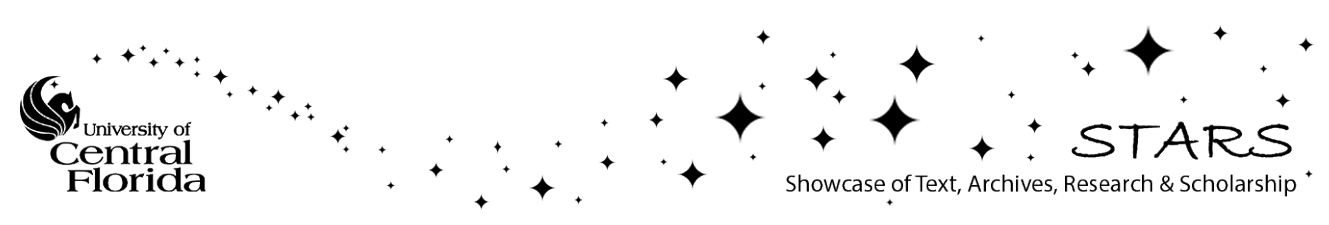




\section{Electrically tunable liquid-crystal photonic crystal fiber}

Cite as: Appl. Phys. Lett. 85, 2181 (2004); https://doi.org/10.1063/1.1796533

Submitted: 04 March 2004 . Accepted: 26 July 2004 . Published Online: 24 September 2004

Fang Du, Yan-Qing Lu, and Shin-Tson Wu

\section{ARTICLES YOU MAY BE INTERESTED IN}

Magneto-optical fiber sensor based on bandgap effect of photonic crystal fiber infiltrated with magnetic fluid

Applied Physics Letters 101, 241118 (2012); https://doi.org/10.1063/1.4772017

Fabrication of functional microstructured optical fibers through a selective-filling technique Applied Physics Letters 85, 5182 (2004); https://doi.org/10.1063/1.1828593

Temperature effect on liquid crystal refractive indices

Journal of Applied Physics 96, 19 (2004); https://doi.org/10.1063/1.1757034

\section{Applied Physics Letters}

Mid-IR and THz frequency combs special collection

\section{Read Now!}




\title{
Electrically tunable liquid-crystal photonic crystal fiber
}

\author{
Fang Du, Yan-Qing Lu, and Shin-Tson Wua) \\ College of Optics and Photonics, University of Central Florida, Orlando, Florida 32816
}

(Received 4 March 2004; accepted 26 July 2004)

\begin{abstract}
Tunable light switch using a photonic crystal fiber filled with nematic liquid crystal is demonstrated. The original band-gap-guiding fiber structure was transformed to a total internal reflection-guiding photonic crystal fiber by filling liquid crystal into the air core and cladding air holes. By applying external voltage to the liquid-crystal-filled fiber, we have demonstrated an electrically tunable fiber-optical switch with over $30 \mathrm{~dB}$ attenuation at $60 \mathrm{~V}_{\mathrm{rms}}$ for a He-Ne laser beam. This liquid-crystal-filled photonic crystal fiber will find useful applications in fiber-optic communication systems. (C) 2004 American Institute of Physics. [DOI: 10.1063/1.1796533]
\end{abstract}

Photonic crystal fiber (PCF) is a microstructured waveguide that contains an array of air holes running down the length of fiber. The air-hole lattice structure provides PCFs an additional design freedom offering different light propagation properties by choosing air-hole periodic structure topology and structural parameters like pitch, core diameter, and air-hole filling. In general, PCFs guide light either by a total internal reflection (TIR) or photonic band-gap (PBG) effect, depending on the fiber structure. For the TIR-guiding fibers, ${ }^{1}$ the refractive index of the core is higher than that of cladding. Therefore, the guiding mechanisms are similar to conventional fibers. In the case of PBG fiber, ${ }^{2}$ light of a certain wavelength bandwidth is confined in the low-index core by a full two-dimensional photonic band-gap effect. The air-hole lattice structure not only provides more design freedom, but also broadens the potential applications of PCFs by introducing additional materials into the air holes. ${ }^{3-6}$ Among these materials, liquid crystal (LC) is of particular interest because its refractive index can be tuned by temperature or by electric field. Some LC photonic band-gap composite structures ${ }^{7-9}$ and slab waveguides ${ }^{10}$ have been demonstrated by tuning the temperature. Recently, Larsen and coauthors reported a LC photonic band-gap fiber for optical switch using thermooptical tuning. ${ }^{11}$

In this letter, we report the experimental observations of an electrically tunable LC-filled index-guiding photonic crystal fiber. The output light intensity of such a fiber can be electrically tuned by applying an external voltage across the fiber. We have investigated the electro-optic properties of such a fiber and demonstrated the switching function of over $30 \mathrm{~dB}$ attenuation at $\sim 60 \mathrm{~V}_{\mathrm{rms}}$. In addition, the fiber transmittance is dependent on the input laser polarization in the intermediate voltage regime. Good attenuation in the high voltage state is, however, independent of the input polarization.

The PCF we used in the experiment was manufactured by BlazePhotonics. The microscope picture of the PCF structure is shown in Fig. 1(a). The fiber has a 10.9- $\mu \mathrm{m}$ diameter air core and a microstructured cladding containing an array of air holes in undoped fused silica along the length of the fiber. The pitch (distance between cladding hole centers) is $3.8 \mu \mathrm{m}$ and the diameter of the microstructured silica-holes region is $\sim 70 \mu \mathrm{m}$. There is an additional cladding layer of

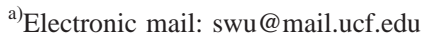

pure silica surrounding the microstructured region, giving the total fiber diameter of $120 \mu \mathrm{m}$. Because the fiber is designed for $\lambda=1.55 \mu \mathrm{m}$, it does not guide other wavelengths. Figure 1(b) shows the charge coupled device (CCD) camera picture of the fiber output when a He-Ne laser is used as a light source. From the picture, one can see that light distributes in the entire holey region and is not guided in the fiber core.

We removed the fiber-protective jacket and filled a 12-mm-long air-core PCF with a commercial Merck nematic LC mixture E48 $(\Delta n=0.23$ at $\lambda=589 \mathrm{~nm})$ by capillary action. Because there is no aligning pretreatment, the alignment of LC molecules in the core and cladding holes is highly affected by the interface between the LC and the fiber holes. According to previous studies, ${ }^{12-14}$ the nematic LC directors tend to align along the length of the fiber in silica or Pyrex capillaries. In order to examine the director orientation and uniformity of LC alignment, the E48-filled PCF sample was placed under a polarizing optical microscope. The fiber was oriented parallel to the microscope polarizer. In the crossed analyzer-polarizer configuration, even though it was barely visible when observed by naked eyes through the microscope, a small amount of light in the microstructed region was captured by the attached camera, shown as inset (a) in Fig. 2. Therefore, we conclude that the LC director alignment is mainly along the fiber axis, in agreement with other reports, ${ }^{12-14}$ but with a small tilt resulting in a slight light rotation.

Figure 2 shows the experimental setup for characterizing the electro-optical properties of the E48-filled PCF. The fiber was fixed between two parallel flat glass substrates with indium-tin oxide (ITO) deposited on the inner surfaces and voltage applied to the fiber as shown in inset (b) of Fig. 2. A

(a)
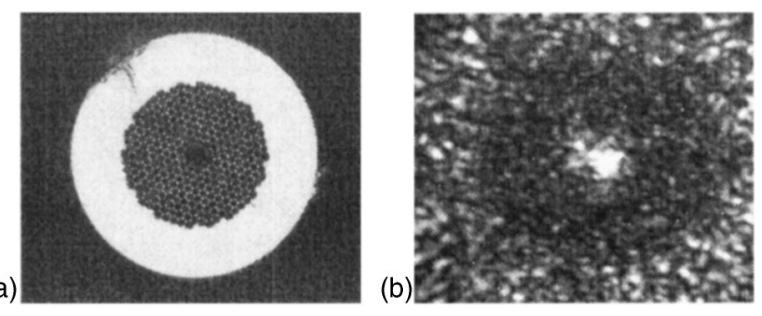

FIG. 1. Pictures of BlazePhotonics band-gap PCF: (a) microscope picture of the fiber structure and (b) CCD camera picture of the fiber output of the He-Ne laser beam. $\lambda=633 \mathrm{~nm}$ and $T=23{ }^{\circ} \mathrm{C}$. 


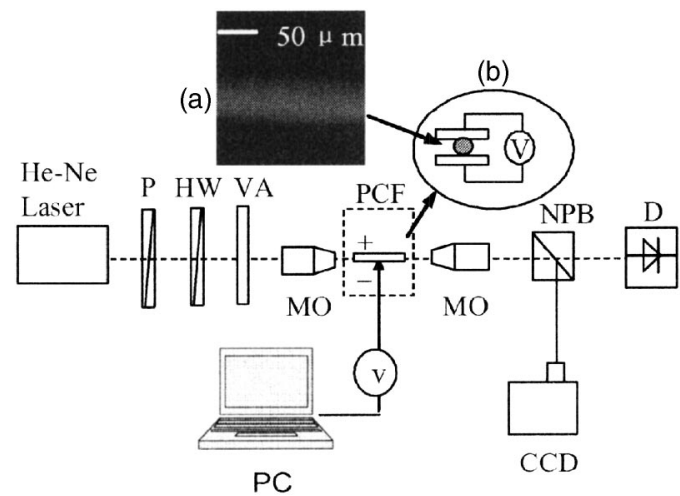

FIG. 2. Experimental setup for characterizing the electro-optic properties of the E48-filled PCF. $\mathrm{P}=$ polarizer, $\mathrm{HW}=$ half-wave plate, $\mathrm{VA}=$ variable attenuator, $\mathrm{MO}=$ microscope objective, $\mathrm{PCF}=$ photonic crystal fiber, NPB $=$ nonpolarizing beamsplitter, $\mathrm{D}=$ detector. $\lambda=633 \mathrm{~nm}$ and $T=23{ }^{\circ} \mathrm{C}$.

linearly polarized He-Ne laser with polarization parallel to the applied electric field was used as a probe light. The sample was fixed on a high-precision XYZ stage and positioned between two $10 \times$ microscope objectives (MO). The first objective lens focused the input beam to a $10 \mu \mathrm{m}$ spot and coupled it to the PCF core. The second MO served to outcouple the laser beam from the fiber and then image the output beam profile onto a CCD camera. A computercontrolled LABVIEW system and voltage amplifier were used to provide voltage to the fiber. A nonpolarizing beam splitter was used to split the output beam from the fiber to the CCD camera and photodiode detector.

Figure 3 depicts a sequence of the CCD camera pictures of the E48-filled PCF output at different applied voltages. At $V=0$, the fiber core clearly guides the laser light of Gaussian profile. In a simplified picture we could approximate the mi-
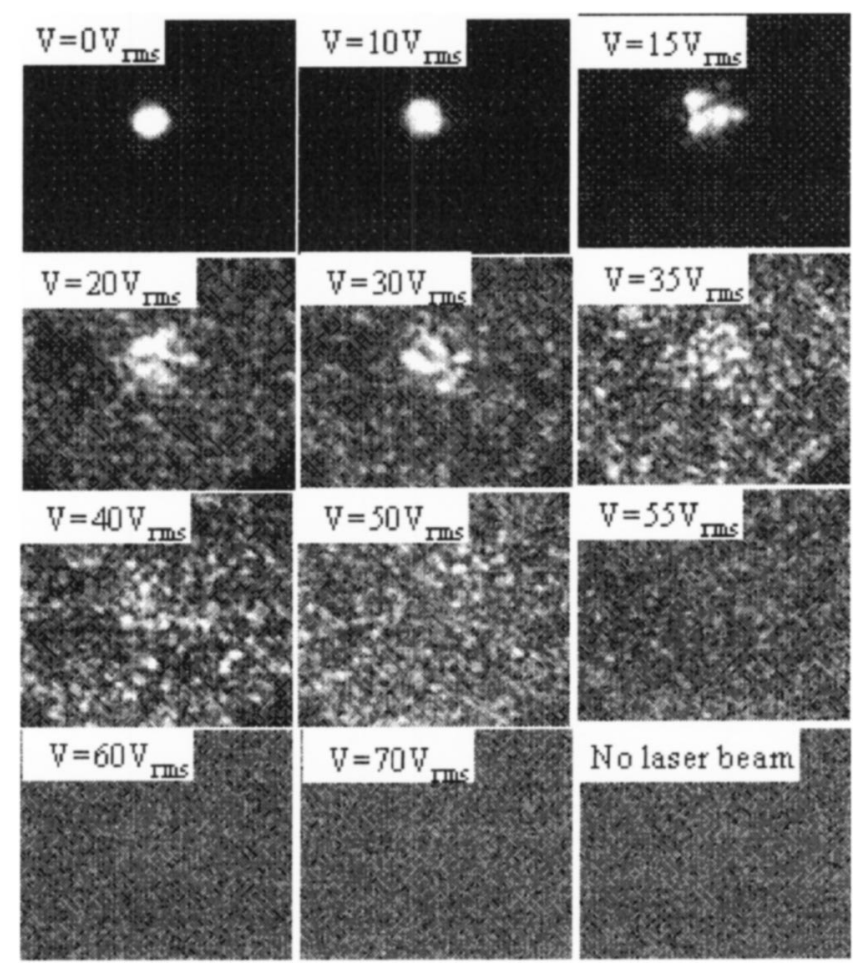

FIG. 3. CCD camera pictures of the E48-filled PCF output at different voltages. The laser polarization is parallel to the direction of electrical field. $\lambda=633 \mathrm{~nm}$ and $T=23{ }^{\circ} \mathrm{C}$.

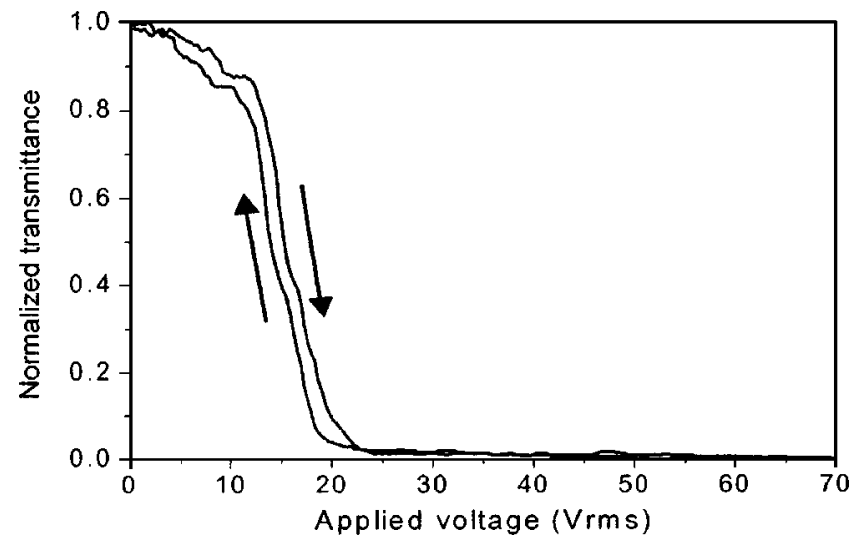

FIG. 4. The voltage-dependent normalized transmittance of PCF filled with E48. The laser polarization is parallel to the applied field direction. $\lambda$ $=633 \mathrm{~nm}$ and $T=23{ }^{\circ} \mathrm{C}$.

crostructured region as consisting of the core filled with LC and the uniform cladding of the effective refractive index. Due to the higher refractive index of the LC E48 compared to that of the silica, the fiber core has a higher refractive index than the "effective" cladding that consists of $10 \%$ silica (index of refraction 1.45) and 90\% E48 (in the initial directors orientation configuration refractive index $\sim 1.6$ ). The guiding mechanism of the original air-core PCF switched from the original PBG effect to TIR guiding (also called index guiding) after it was filled with E48. In addition, the experiment showed that the fiber supports multimodes. If the laser beam was not coupled into the fundamental mode, then the output beam profile was irregular and less Gaussianlike. This multimode nature of the fiber, for the given experimental parameters, was further confirmed by the finite difference technique (FDT) simulations.

As the applied voltage increases, the core-guided mode loses the symmetry and Gaussian profile, becomes irregular in shape, drops in the peak intensity, and gradually couples more and more to the cladding LC-filled holes instead of the core itself, as shown in Fig. 3. From the CCD camera pictures one can see this occurring at $V>5 \mathrm{~V}_{\text {rms. At }} V$ $\sim 20 \mathrm{~V}_{\mathrm{rms}}$, the beam propagation is mainly confined to the "cladding holes" region, especially in those adjacent holes surrounding the core, and the light intensity in the core region is very weak. The light propagating through the cladding regions contains a number of small bright spots, indicating "guiding" by the LC-filled cladding holes. Once the voltage is further increased to $60 \mathrm{~V}_{\mathrm{rms}}$, the guided throughput signal is reduced dramatically and obscured by the CCD camera noise. In this case, the light propagates not even in the cladding holes region, but rather, in the pure silica cladding region that surrounds the PCF structure. In this highvoltage regime, the light eventually leaks out of the fiber.

In order to quantitatively characterize the switching properties of the index-guided E48-filled PCF, a photodiode detector was placed in the image plane of the fiber output. The light entering the detector was limited by an aperture to capture the light coming from the core only. However, in practice, a fraction of the cladding "modes" might be still detected by the detector causing a small fluctuation in the measurements. The voltage-dependent transmittance of the fiber output with forward and backward voltage scan is shown in Fig. 4, where the transmittance is normalized to the initial transmission in the voltage-off state. At zero voltage, 
the laser beam is mainly guided in the LC core. As the voltage is increased, the guided light gradually leaks to the outer LC-filled holes. As a result, the transmittance is rapidly decreased. The dynamic range was measured to be over $30 \mathrm{~dB}$ at $60 \mathrm{~V}_{\text {rms. }}$. When the voltage was scanned backward from 70 to $0 \mathrm{~V}_{\mathrm{rms}}$, the transmittance curve followed closely to that of the forward voltage scan except for a relatively small hysteresis $\sim 1.3 \mathrm{~V}_{\text {rms }}$. Here, the hysteresis width is defined as $V_{\text {down }}-V_{\text {up }}$, where $V_{\text {down }}$ and $V_{\text {up }}$ are the voltages corresponding to $50 \%$ transmittance for the forward and reverse voltage scans. The switching property is well reproducible. The rise and decay times were measured at $60 \mathrm{~V}_{\text {rms }}$ and $T \sim 23{ }^{\circ} \mathrm{C}$ to be $\sim 10$ and $200 \mathrm{~ms}$, respectively. These results are consistent with those estimated from the 10.9- $\mu$ m diameter E48 LC-filled PCF core. To improve the response time, we could select a LC mixture with small viscoelastic coefficient, reduce the fiber core diameter, operate the device at an elevated temperature, or use the overdrive and undershoot voltage effect. ${ }^{15}$

The light leakage behavior observed in Fig. 3 could originate from the disturbance of the guided mode resulting from the nonuniform reorientation of the LC molecules in the voltage-on state. From polarizing microscopy, the LC molecules in the core and the cladding holes show relatively uniform behavior down the whole fiber length with the applied voltage. However, experimentally, it is very difficult to precisely determine how LC molecules rotate in the voids when the voltage is applied. The interactions between LC molecules and fiber capillaries make the electric field-LC molecules system more complex. From the fiber structure viewpoint, the optical switching behavior is a consequence of efficient coupling between the LC core and the surrounding LC-filled holes. The size of the cladding holes is $3.8 \mu \mathrm{m}$, which is much bigger than the He-Ne laser wavelength $(\lambda$ $=633 \mathrm{~nm}$ ), allowing the possibility for "guiding" within these LC-filled cladding holes.

We also investigated the electro-optic properties of the E48-filled PCF when the input laser polarization was orthogonal to the applied electric field. The results are similar to those of the parallel case. In the null voltage stage the beam was efficiently guided. With the increasing voltage the guiding was quite rapidly lost, first to the cladding holes region, and finally, to the pure silica region. The voltagedependent transmittance is plotted in Fig. 5. The results are slightly different from those shown in Fig. 4. The guided light has a higher threshold voltage as observed from the insets of Fig. 5. The transmittance remains flat at $V$ $\sim 10 \mathrm{~V}_{\text {rms }}$, which shows that the ordinary wave has a higher threshold voltage. This phenomenon is similar to that observed in a LC Fabry-Perot cavity. ${ }^{16}$ In the ideal case, when there was no scrambling of the LC molecules orientation, the horizontal polarization would not see any change in the refractive index even if the voltage is applied. However, as we observed from the polarizing microscope that the LC directors are not aligned completely parallel to the fiber axis. For the 12-mm-long fiber, even a small tilt angle in the LC molecules would induce a noticeable polarization rotation after the light has traversed such a long distance. As a matter of fact, the LC alignment in the fiber holes can be more complicated due to the interactions of the LC molecules with the

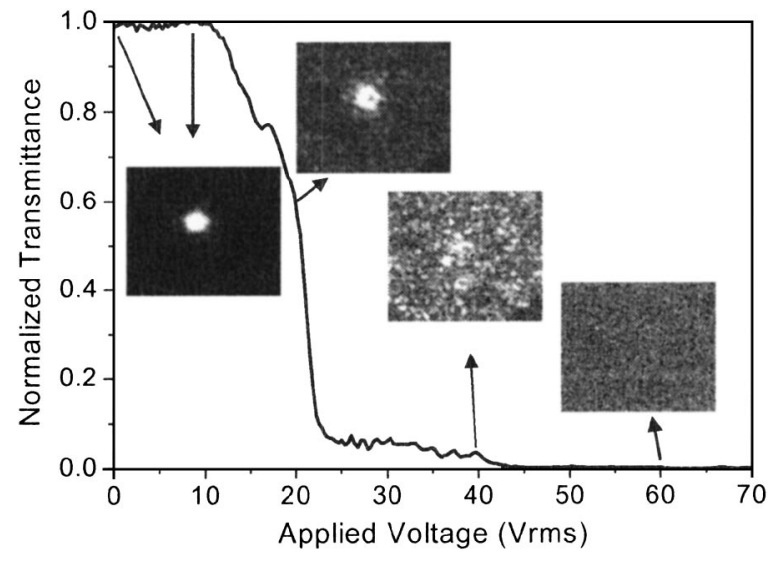

FIG. 5. The voltage-dependent normalized transmittance of PCF filled with E48. The insets show the CCD camera pictures corresponding to different applied voltages. The laser polarization is perpendicular to the electric-field direction. $\lambda=633 \mathrm{~nm}$ and $T=23{ }^{\circ} \mathrm{C}$.

interface of the fiber holes. The detailed LC alignment effect in the PCF, and the associated electro-optic properties, need to be studied further.

In conclusion, by infiltrating LC mixture into an air-core band-gap PCF we achieved an index-guiding PCF configuration. In this index-guiding PCF we have demonstrated an electrically controllable optical switch by applying voltage on the fiber. The disturbance resulting from the electric-fieldinduced LC reorientation leads to a very dramatic and rapid vanishing of the fiber core guiding. The achieved optical attenuation is over $30 \mathrm{~dB}$ at $\sim 60 \mathrm{~V}_{\text {rms }}$ for the 12-mm-long LC-filled PCF. Useful applications in the fiber-optic systems are foreseeable.

The authors are indebted to Dr. Tom Wu, Dr. Yuhua Huang, and Dr. Xinyu Zhu for their technical assistance and discussions. This work is supported by AFOSR under Contract No. F49620-01-1-0377.

${ }^{1}$ J. C. Knight, T. A. Birks, P. St. J. Russell, and D. M. Atkin, Opt. Lett. 21, 1547 (1996).

${ }^{2}$ J. C. Knight, J. Broeng, T. A. Birks, and P. St. J. Russell, Science 283, 1476 (1998).

${ }^{3}$ P. S. Westbrook, B. J. Eggleton, R. S. Windeler, A. Hale, T. A. Strasser, and G. L. Burdge, IEEE Photonics Technol. Lett. 12, 495 (2000).

${ }^{4}$ F. Benabid, J. C. Knight, and P. St. J. Russell, Opt. Express 10, 1195 (2002).

${ }^{5}$ F. Benabid, J. C. Knight, G. Antonopoulos, and P. St. J. Russell, Science 298, 399 (2002)

${ }^{6}$ Y. L. Hoo, W. Jin, H. L. Ho, and D. N. Wang, IEEE Photonics Technol. Lett. 15, 1434 (2003).

${ }^{7}$ K. Busch and S. John, Phys. Rev. Lett. 83, 967 (1999).

${ }^{8}$ K. Yoshino, Y. Shimoda, Y. Kawagishi, K. Nakayama, and M. Ozaki, Appl. Phys. Lett. 75, 932 (1999).

${ }^{9}$ S. W. Leonard, J. P. Mondia, H. M. Driel, O. Toader, S. John, K. Busch, A. Birner, U. Gosele, and V. Lehmann, Phys. Rev. B 61, R2389 (2000).

${ }^{10}$ Ch. Schuller, F. Klopf, J. P. Reithmaier, M. Kamp, and A. Forchel, Appl. Phys. Lett. 82, 2767 (2003).

${ }^{11}$ T. T. Larsen, A. Bjarklev, D. S. Hermann, and J. Broeng, Opt. Express 11, 2589 (2003).

${ }^{12}$ Y. Jeong, B. Yang, B. Lee, H. S. Seo, S. Choi, and K. Oh, IEEE Photonics Technol. Lett. 12, 519 (2000).

${ }^{13}$ M. Green and S. J. Madden, Appl. Opt. 28, 5202 (1989).

${ }^{14}$ I. C. Khoo, Liquid Crystals (Wiley, New York 1994).

${ }^{15}$ S. T. Wu and D. K. Yang, Reflective Liquid Crystal Displays (Wiley, New York 2001).

${ }^{16}$ Y. Huang, T. X. Wu, and S. T. Wu, J. Appl. Phys. 93, 2490 (2003). 